\section{Likelihood Ratio, positives}

\author{
R.-D. Hilgers ${ }^{1}$, N. Heussen ${ }^{1}$ und S. Stanzel ${ }^{2}$ \\ ${ }^{1}$ Institut für Medizinische Statistik, Universitätsklinikum der \\ RWTH Aachen, Aachen, Deutschland \\ ${ }^{2}$ DKFZ Heidelberg, Heidelberg, Deutschland
}

Synonym(e) Likelihood Ratio für ein positives Testergebnis; Likelihood-Quotient; LR+

Englischer Begriff positive likelihood ratio

Definition Das positive Likelihood Ratio ist definiert als der Quotient von Sensitivität und (1-Spezifität) ( $\triangleright$ Sensitivität, diagnostische; $>$ Spezifität, diagnostische).

Beschreibung Das positive Likelihood Ratio (LR+) beschreibt das Verhältnis der Wahrscheinlichkeit für ein positives Testergebnis unter den Erkrankten zur Wahrscheinlichkeit für ein positives Testergebnis unter den Gesunden $(\triangleright$ Testergebnis, falsch-positives). Es gibt an, wie wahrscheinlich es ist, ein positives Testresultat bei einer erkrankten Person zu finden, im Verhältnis zur Wahrscheinlichkeit, ein positives Testresultat bei einer nicht erkrankten Person zu finden. Entsprechend der Definition wird das LR+ geschätzt durch den Quotienten (a / $(\mathrm{a}+\mathrm{c})) /(1-\mathrm{d} /(\mathrm{b}+\mathrm{d}))$ (Bezeichnungen $>$ Vierfeldertafel, s. Tabelle). Likelihood Ratios sind unabhängig von der $>$ Prävalenz. Sie lassen sich auch für Gruppen definieren, die in mehr als 2 Kategorien vorliegen. Anhand der Likelihood Ratios lässt sich - basierend auf den Ergebnissen des $\$ A priori odds - das - A posteriori odds ermitteln. Insofern beschreibt das Likelihood Ratio den Informationsgewinn gegenüber dem A-prioriWissen, wenn ein positives Testergebnis vorliegt. Das Likelihood Ratio entspricht der Steigung der $>$ ROC-Kurve pro Schwellenwert ( $\triangleright$ Schwellenwert der ROC). Formal lässt sich das A posteriori odds durch Multiplikation des A priori odds mit dem positiven Likelihood Ratio berechnen. Dies mag der Grund dafür sein, dass man in der Literatur häufig nur das positive Likelihood Ratio bespricht und dieses auch kurz „Likelihood Ratio“ nennt.

\section{Literatur}

Hilgers R-D, Bauer P, Scheiber V (2002) Einführung in die Medizinische Statistik. Springer, Berlin/Heidelberg/New York 